\title{
Production Planning and Scheduling Optimization Model: A case of study for a Glass Container Company
}

\author{
Laura Hervert-Escobar \\ and Oscar A. Esquivel-Flores \\ Instituto Tecnológico de Estudios Superiores de Monterrey \\ Monterrey, Nuevo Leon, Mexico
}

\begin{abstract}
Based on a case study, this paper deals with the production planning and scheduling problem of the glass container industry. This is a facility production system that has a set of furnaces where the glass is produced in order to meet the demand, being afterwards distributed to a set of parallel molding machines. Due to huge setup times involved in a color changeover, manufacturers adopt their own mix of furnaces and machines to meet the needs of their customers as flexibly and efficiently as possible. In this paper we proposed an optimization model that maximizes the fulfillment of the demand considering typical constraints from the planning production formulation as well as real case production constraints such as the limited product changeovers and the minimum run length in a machine. The complexity of the proposed model is assessed by means of an industrial real life problem.
\end{abstract}

\section{INTRODUCTION}

Currently, the activities of the planning and control of companies are becoming increasingly complex. The managers of this area are constantly pressured to reduce operating costs, maintain inventories at adequate levels, to fulfill the demand of customers, and to respond effectively to changes. There are plenty tools that help to meet these goals, however, they do not consider real factors that have and impact in the result, consequently, additional decisions should be make to complete the process.

\section{PRoblem Definition}

Consider a manufacturing system that produces glass containers. The process begins with the mixtures of raw materials which determines the color of the glass, this mixture includes the recycling glass. The mixture is transported into a furnace where it is melted. The glass paste is fed to a set of parallels glass moulding machines $m$ that shape the product. The formed containers are then passed through a strict quality inspection. Containers found to be defective are discarded and melted down in the furnace as 'cullet'. Once they have been quality approved, the containers are packed on pallets at the end of the product lines. If it is required, the product pass through a decoration process, otherwise the product is stored in the warehouse or shipped for sale.

\section{MATHEMATICAL SECTION}

Mathematical formulation of the model is defined in the following equations. Objective function given in Equation 1 maximizes the benefits of the fulfillment of demand $v_{t}^{j}$ by penalizing the unsatisfied demand $f_{j}^{t}$. Thus, the cost $h$ of not being able to satisfied the demand is the same for all shapes while the benefit is differentiated first by SKU $\hat{g}_{s}$ and consequently by shape $g_{j}=\sum_{j(s)} \hat{g}_{s}$. Additionally, a normalized cost $a_{j, m}$ of production is considered. This cost is associated with the performance of the combination of product-machine $x_{j, m}^{t}$. Shapes and SKU's are interrelated, where a subset of SKU's can be obtained from one type of shape. In this way from each $j$ there are $n$ products, we will refer the number of products generated by some shape $j$ as $j(s)$.

$\operatorname{Maximize}(z)=\sum_{J} \sum_{T} g_{j} v_{j}^{t}-\sum_{J} \sum_{M} \sum_{t} a_{j, m} x_{j, m}^{t}-\sum_{J} \sum_{T} h f_{j}^{t}$

Constraints for this model are as follows:

$$
x_{j, m}^{t} \leq 1 \quad \forall j \in J, m \in M, t \in T
$$

A machine $m$ can produce only one type of shape $j$ during period $t$ this condition is checked in Equation 2 .

$$
x_{j, m}^{t} \leq b_{j, m}^{t} \quad \forall j \in J, m \in M, t \in T
$$

Therefore, the availability of a machine for a certain product in period $t$ according with a given timetable $b_{j, m}^{t}$ is outlined in Equation 3. This constraint allows to prove different campaigns of color in the furnaces. Also, it is helpful to define a maintenance program for the planning horizon. During this case study, the minimum run length per machine allowed is three days. This condition is given in Equations 4 to 6 .

$$
\begin{aligned}
x_{j, m}^{t-1}+x_{j, m}^{t+1} \geq x_{j, m}^{t} & \forall j \in J, m \in M, t=2, \ldots,|T|-1 \\
x_{j, m}^{t-2}+x_{j, m}^{t+1} \geq x_{j, m}^{t-1} & \forall j \in J, m \in M, t=3, \ldots,|T|-1 \\
x_{j, m}^{t-2}+x_{j, m}^{t} \geq x_{j, m}^{t-1} & \forall j \in J, m \in M, t=3, \ldots,|T|(6)
\end{aligned}
$$


Staff for changeovers is typically a team of highly skill workers. Therefore, this resource is limited in the plant. Also, due to the time required to change the product and stabilize the process, the changeover $c_{j, m}^{t}$ are limited to one during weekdays.

$$
\begin{gathered}
x_{j, m}^{t}-x_{j, m}^{t-1} \leq c_{j, m}^{t} \quad \forall j \in J, m \in M, t=2, \ldots,|T| \\
x_{j, m}^{t}-x_{j, m}^{t-1} \leq-1+2 * c_{j, m}^{t} \quad \forall j \in J, m \in M, t=2, \ldots,|T| \\
\sum_{J} \sum_{M} c_{j, m}^{t} \leq 1 \quad \forall t \in W \\
\sum_{J} \sum_{M} c_{j, m}^{t}=0 \quad \forall t \in \hat{W}
\end{gathered}
$$

Equations 7 and 8 allow to identify the day of a changeover in a machine. While, Equations 9 and 10 limits to one changeover product in the whole plant during the weekdays, and prevent changes during the weekend. The production $p_{j, m}^{t}$ is given by the rate $r_{j, m}$ of the selected machine, however, when a change of product happens, the rate decreases $25 \%$, and this condition is given in Equation 11.

$r_{j, m} * x_{j, m}^{t}-0.25 * r_{j, m} * c_{j, m}^{t}=p_{j, m}^{t}$

$\forall j \in J, m \in M, t \in T 55$

Inventory balance equation is given in Equation 12. The balance of satisfied $\left(v_{j}\right)$ and unsatisfied $\operatorname{demand}\left(f_{j}\right)$ is given in Equation 13.

$$
\begin{aligned}
\sum_{M} p_{j, m}^{t}+i_{j}^{t-1}=i_{j}^{t}+v_{j}^{t} & \forall j \in J, t \in T, i_{j}^{0}=y_{j}^{0} \\
v_{j}^{t}+f_{j}^{t}=d_{j}^{t} & \forall j \in J, t \in T
\end{aligned}
$$

Inventory $i_{j}^{t}$ is bounded by two situations, first, by the inventory policy of the SKU, given in Equation 14, and second by the capacity of the warehouse, given in Equation 15.

$$
\begin{gathered}
i_{j}^{t} \leq u_{j} \quad \forall j \in J, t \in T \\
\sum_{J} i_{j}^{t} \leq q \quad \forall t \in T
\end{gathered}
$$

\section{Solution Methods}

Glass container sales are highly seasonable and variable. The products are classified into three categories: A, B, C regarding the variability and the volume of the demand. Furnaces handle only one color at the time. The color and availability of the furnaces and machines is given in a predefined calendar. The first furnace (FA) has a capacity of 260 ton and has two parallel molding machines $\left(m_{1}, m_{2}\right)$ to shape the product. Second furnace (FB) has a capacity of 360 ton and has three

\begin{tabular}{|c|c|c|c|c|c|c|c|c|c|c|}
\hline \multirow{2}{*}{ ID } & \multicolumn{2}{|c|}{ \% Demand | } & \multicolumn{3}{|c|}{ ID } & \multirow{2}{*}{ WH } & \multirow{2}{*}{$\mathrm{CO}$} & \multicolumn{2}{|c|}{ RL (days) } & \multirow{2}{*}{ ET } \\
\hline & $\begin{array}{l}\text { Sat- } \\
\text { is- } \\
\text { fied }\end{array}$ & $\begin{array}{l}\text { Un- } \\
\text { sat- } \\
\text { is- } \\
\text { fied }\end{array}$ & $\mathbf{A}$ & B & $\mathbf{C}$ & & & MIN & $\overline{\text { MAX }}$ & \\
\hline E0 & \multicolumn{2}{|c|}{$98.99 \% 1.01 \%$} & 14 & 46 & 66 & $88 \%$ & 186 & 3 & 151 & 0.67 \\
\hline E1 & \multicolumn{2}{|c|}{$99.15 \% 0.85 \%$} & 13 & 49 & 60 & $86 \%$ & 204 & 3 & 202 & 2.7 \\
\hline E2 & \multicolumn{2}{|c|}{$99.00 \% 1.00 \%$} & 14 & 52 & 53 & $89 \%$ & 186 & 3 & 275 & 1.14 \\
\hline E3 & \multicolumn{2}{|c|}{$99.16 \% 0.84 \%$} & 16 & 52 & 105 & $126 \%$ & 211 & 3 & 180 & 0.83 \\
\hline E4 & \multicolumn{2}{|c|}{$99.57 \% 0.43 \%$} & 14 & 48 & 65 & $77 \%$ & 198 & 3 & 175 & 4.12 \\
\hline E5 & \multicolumn{2}{|c|}{$99.39 \% 0.61 \%$} & 10 & 42 & 70 & $67 \%$ & 183 & 3 & 312 & 2 \\
\hline E6 & \multicolumn{2}{|c|}{$99.12 \not 0.88 \%$} & 13 & 52 & 57 & $87 \%$ & 197 & 3 & 182 & 16.2 \\
\hline E7 & \multicolumn{2}{|c|}{$98.68 \% 1.32 \%$} & 26 & 62 & 83 & $89 \%$ & 186 & 3 & 268 & 12 \\
\hline
\end{tabular}
parallel molding machines $\left(m_{3}, m_{4}, m_{5}\right)$ to shape the product. Finally, the third furnace (FC) also has a capacity of 360 ton but has 4 molding parallel machines to shape the product $\left(m_{6}, m_{7}, m_{8}, m_{9}\right)$. The molding machines are able to produce 55 types of shapes or sub-products that are later turned into 135 types of final products (SKU's). This transformation takes place in the decoration process, which has unlimited capacity.
TABLE I: Business metrics results

Several scenarios were designed according to the needs of the company. The scenarios were designed under different inventory policies for products A and B which represent $95 \%$ of demand. Products under classification $\mathrm{C}$ are Make-To-Order and do not have inventory policy. The horizon planning is for 7550 days with a bucket of one day.The scenario E0 use the history data of the company. The rest of the scenarios use a forecast demand for the following 550 days. Then, scenario E1 helps to find a solution in case the forecast demand is actually 5\% higher. Scenario E2 tests the forecast demand with a change in the upper bound of the mix of products. Scenario E3 considers an unlimited capacity in the warehouse, in the case of study, the company has a limited space for store, this scenario analyzes the result of the metrics of interest when such limitation does not exist. Another situation of interest in this case of study is when the current capacity of the warehouse is reduced by external situations. Then, scenario E4 tests a reduction of $12.5 \%$ of the capacity and E5 considers a $25 \%$ of reduction. Scenario E6 tests the reduction of the upper bound for the products make-to-stock, or with classification A and B. Finally, scenario E7 tests the stop of operations at the end of the year.

The proposed model was implemented using AMPL to call the optimizer GUROBI 5.6. The results of the proposed methodology are given in terms of business metrics and the execution time.

\section{REsults}

The results for business metrics are showed in table 1 . Where rows are used to identify the scenario and columns denote the business metric of interest. The table provides the result for each scenario and business metric. The business metrics of interest are the service level given by the satisfied and unsatisfied demand, the average inventory days $I D$ for each type of product, the final inventory level, which is given as a percentage of utilization of the warehouse, the product changeovers in a year $C O$, and the minimum and maximum run-length $R L$ of a product, and the execution time $E T$ of the model. 
As table 1 shows, most of the scenarios improved the service level in comparison with the current result. Due to the data confidentiality, it is not possible to present the real numbers of production and benefits. Although the improvement in terms of service level seems small, the results in economic benefits and the utilization of the warehouse fully justify the implementation of the tool. The solving time increases significantly as the capacity of the production plant and/or the warehouse is reduced. The proposed model generates approximately 82,000 continuous variables and 110,000 integer variables. But even in those cases, the model is able to achieve an optimal solution within a reasonable time for the user of the tool. It is expected that the company uses the tool at most once per month and at least once per semester in order to readjust strategies.

\section{CONCLUSIONS}

This paper presents an approach to solve a real life production planning and scheduling problem. The model represents as possible the operation of a glass container industry. In this research, we provide a tool that provides a strategy for the planning production as well as detailed information (scheduling plan) to apply the proposed strategy. The fact that we are deriving long-term plans might reduce the importance of the computation times. The tool is flexible enough to test different scenarios under a reasonable time according to the availability of the final user. Next step in this research is to include the scheduling of the color campaign in the furnaces and the load of product machines simultaneously. This will lead to ability to analyze different approaches for the solution since the size and complexity of the problem will increase significantly.

\section{ACKNOWLEDGEMENTS}

This research has been submitted for presentation at CLAIO 2016. The authors are grateful to Sintec for financial and technical support during the development of this research. Sintec is the leading business consulting firm for Supply Chain, Customer and Operations Strategies with a consultative model in Developing Organizational Skills that enable their customers to generate unique capabilities based on processes, organization and IT. Also, we appreciate the financial support of CONACYT-SNI program in order to promote quality research.

\section{REFERENCES}

[1] J. F.; Caravilla M. A. Almada-Lobo, B.; Oliveira. Production planning and scheduling in the glass container industry: A vns approach. International Journal of Production Economics, 114(1):363-375, 2008.

[2] Michael O. Ball. Heuristics based on mathematical programming. Surveys in Operations Research and Management Science, 16(1):21 - 38, 2011.

[3] Marcio; Yukio Bressan Hossomi Marcelo;Almada-Lobo Bernardo. Fabiano Motta Toledo, Claudio;da Silva Arantes. Mathematical programmingbased approaches for multi-facility glass container production planning. Computers \& Operations Research, 74:92-107, 2016.

[4] Claudio Fabiano Motta Toledo, MáRcio Da Silva Arantes, Renato Resende Ribeiro De Oliveira, and Bernardo Almada-Lobo. Glass container production scheduling through hybrid multi-population based evolutionary algorithm. Appl. Soft Comput., 13(3):1352-1364, March 2013.
[5] Bernardo Almada-Lobo; Diego Klabjan; Maria Antónia Carravilla; José F. Oliveira. Multiple machine continuous setup lotsizing with sequence-dependent setups. Computational Optimization and Applications, 47(3):529-552, 2010.

\section{BIO}

\section{Name:}

LAURA

Family Name:

HERVERT-ESCOBAR

Institution

INTITUTO TECNOLOGICO DE ESTUDIOS SUPERIORES DE MONTERREY

\section{Department:}

Research

Group:

Industrial Engineering and numerical methods

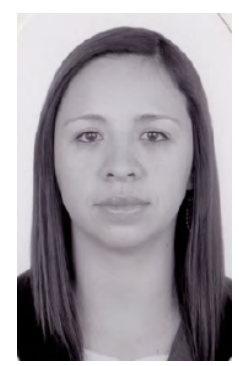

\section{Short Bio:}

(max. 150 words)

Postdoctoral researcher at Instituto Tecnológico de Estudios Superiores de Monterrey, her research include, but are not limited to the development, analysis and implementation of metaheuristic techniques for solving complex real-life combinatorial problems with several objectives. Currently, the principal emphasis will be the study and development of advanced mathematical methods and algorithms both stochastic and hybrid (stochastic/deterministic) ones for Linear Algebra (Matrix Inversion, Solving Large Systems of Linear Equations, etc.) and multi-objective multi-constrained optimization. Also the modeling of complex systems using stochastic and hybrid approaches as well as Network Science techniques, mainly parallel algorithms and parallel computing. Work experience includes the development of different projects including new product launch, design and opening of workshops, business model re-engineering, etc.

Talk/Poster's title:

Production Planning and Scheduling Optimization Model: A case of study for a Glass Container Company 